\title{
'ig,vgs' Monitoring for Fast and Robust SiC MOSFET Short-Circuit Protection with High integration Capability
}

\author{
Yazan Barazi, François Boige, Nicolas Rouger, Jean-Marc Blaquiere, Frédéric Richardeau \\ LAPLACE, University of Toulouse, CNRS, INPT, UPS, Toulouse, France \\ 2 rue Charles Camichel Toulouse P722 - 31071 \\ Toulouse, France \\ Tel.: +33 / (0)5 - 3432.23.91. \\ Fax: $+33 /(\mathrm{x}) 5-6163.88 .75$. \\ barazi@laplace.univ-tlse.fr; francois.boige@gmail.com; rouger@laplace.univ-tlse.fr; \\ blaquiere@laplace.univ-tlse.fr; frederic.richardeau@laplace.univ-tlse.fr; \\ http://www.laplace.univ-tlse.fr/
}

\section{Acknowledgements}

This research work has been supported financially by the French Ministry in higher education, research and innovation.

\section{Keywords}

«Silicon Carbide $(\mathrm{SiC}) »$, «signal processing», «Robust control», «intelligent drive», «Protection device»

\begin{abstract}
SiC MOSFETs have a low short circuit withstand time. To address this challenge, a soft shut down and two original detection methods are proposed in this paper, easily implemented and based-on (ig, vgs) diagnosis with no direct time dependency. The first one is dedicated for SiC MOSFETs using his gate-leakage thermal runaway current, and the second one is more general and faster using the gatecharge monitoring. Both are experimentally validated and compared in terms of response-time and robustness capability.
\end{abstract}

\section{Introduction}

The Silicon Carbide (SiC) MOSFETs offers several advantages, low switching losses, higher switching frequencies and high temperature stability [1]. However, today, the short-circuit (SC) withstanding time $\left(\mathrm{T}_{\mathrm{SC}}\right)$ of SiC MOSFETs is lower than silicon devices one [2], within $t_{S C}=2 \mu s$, [3] instead of $t_{S C}=10 \mu \mathrm{s}$ for Si IGBTs. Power converters designed with these components are therefore less robust. This weakness must be compensated by a protection whose delay must not exceed Tsc/2, as IGBT's standard today, or even less if repeated short-circuit robustness is required.

Power semiconductors devices are exposed to several types of short circuits. In summary, internal Hard switching fault (HSF, SC type1) and external fault under load (FUL, SC type2) are the two main fault behaviors. HSF occurs when the switch turns on with a permanent full bus voltage across itself typically caused by faulted control signals. FUL occurs when the device is already in on-state and an external short-circuit occurs. HSF and FUL can be distinguished by the presence or not of a dv/dt on the drain source of the MOSFETs. In all cases, MOSFETs saturates at high current density leading to a thermal dynamic source of heat in a few microseconds for the least robust components.

Many kinds of SC detection methods have been demonstrated for Silicon IGBTs [4] and SiC MOSFETs [5,6]. Most of the proposed techniques rely on the drain-source voltage or current sensing. Those techniques are more suitable for bipolar transistors such as IGBTs, and on the other hand they are limited due to the use of a high-voltage rating diode or an additional current sensor. A relatively long blanking time is required in the case of the drain-source voltage detection method in order to 
achieve a stable state after the turn-on sequence [7]. The 2D diagnosis [8] is the best candidate to minimize the timing. Indeed, this family of methods applies during the turn-on sequence and not after, and is time independent. This study presents experimental results of two original detection methods of $\mathrm{SiC}$ MOSFETs under short circuit. Both methods are 2D diagnosis based-on low voltage / signals waveforms $\left(\mathrm{I}_{\mathrm{g}}, \mathrm{V}_{\mathrm{gs}}\right)$. The first detection method is developed, and depends on the unique behavior of SiC MOSFETs by the means of its gate leakage current runaway at high temperature at the on-state sequence. Where the second one is an adapted detection method, which depends on the gate charge variation on the switching cycle, between normal operation and short-mode. Those methods detect as early as possible the SC and turn softly the device off. In the full paper, the first method will be presented in general terms while the second one will be more detailed and will consist of the core of the article.

\section{Behavior of SiC MOSFETs under short-circuit}

Under short circuit fault, the MOSFET SiC presents a very high saturation current caused by the strong electrical field imposed in the channel region to minimize the $\mathrm{R}_{\mathrm{DSON}}$ in normal operation as reported in [1]. The saturation current combined with the high electrical field in the depletion region dissipates an enormous amount of energy in few microseconds. The released heat increased, especially, the gate oxide temperature leading to hot electron injection inside the gate also called Schottky emission [9]. The MOSFET SiC behavior is displayed in Fig.1. Two short-circuit electrical mechanism characteristics are especially interesting in this study located in part A and B. Part A of the figure might behave differently regarding the fault type (I or II) respectively Hard Switch Fault HSF or Fault Under Load FUL. This study is focused in short-circuit under HSF, whose fault dynamic is faster than FUL one. On other hand part B, is a behavior unique to SiC MOSFETs. Therefore, two main characteristics are studied in this paper related to the gate of the power transistor is the absence of miller plateau (part A), and the current injection in the gate also called dynamic gate leakage current (part B). In order to simplify the short-circuit under HSF, Fig.1.(b) presents an illustration of the fig.1 part-A.

(a)

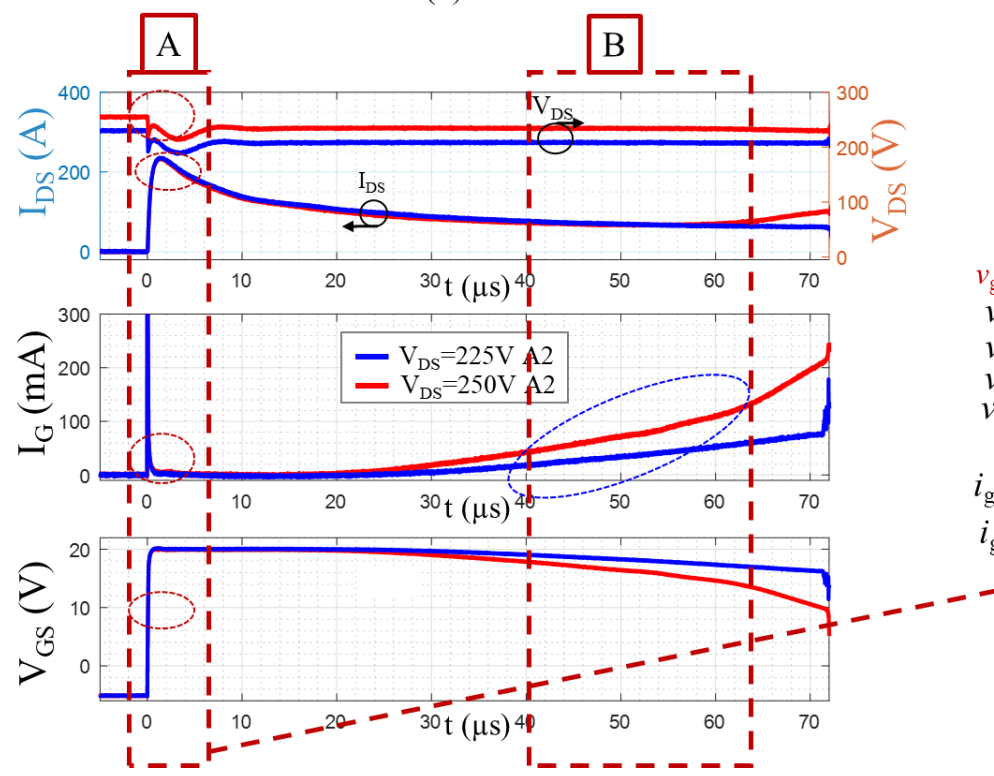

(b)

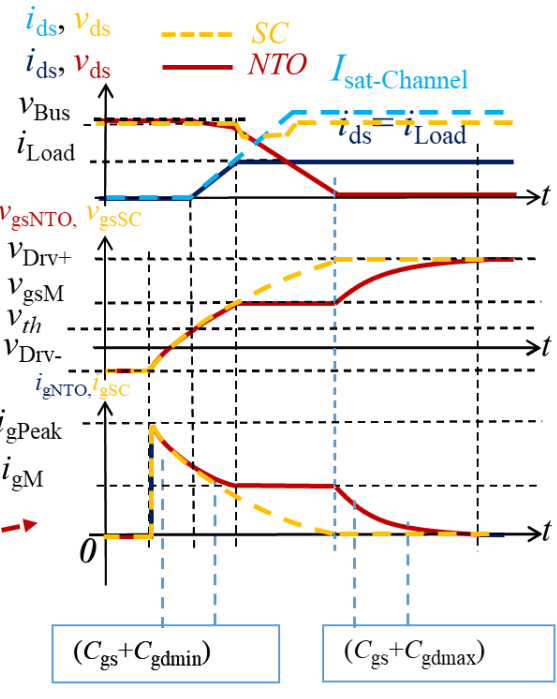

Fig. 1: (a). MOSFET SiC experimental waveforms at two different drain biases under SC, (b).

Simplified illustration between normal-turn on NTO and SC-HSF operations.

DUT : $1.2 \mathrm{kV}, 80 \mathrm{~m} \Omega @ 25^{\circ} \mathrm{C}, \mathrm{R}_{\text {gext }}=47 \Omega, \mathrm{T}_{\text {case }}=25^{\circ} \mathrm{C}$

\section{Detection using the dynamic gate leakage current}

The dynamic gate leakage current can be observed in most MOSFET SiC available today and at about half the devices short-circuit withstanding time (Tsc/2) [10], Fig.1-part A. Thus, detecting this current is a SC marker and, with the appropriate electronics, can lead to a fast shutdown to avoid failure. 
However, detecting a current on the gate can be the normal switching order of device or a current injection through the parasitic Miller capacitance caused by the switching of another device as crosstalk phenomena in inverter leg. The proposed detection method relies on a low current detection and a dedicated logical circuit to sort out the normal operating condition from the short-circuit behavior. The detection circuit principle is presented in fig. 2(a). The current is measured using the external gate resistor as a shunt with an excellent SNR (Signal Noise Ratio) enabling a high robust detection method. The logical circuit uses the PWM switching signal as a reference starting a blanking time of 2 $\mu \mathrm{s}$ to avoid true false positive that is equivalent to Tsc/4 for the device considered.

The proposed detection method has been implemented in an integrated way using SMD components. The logical circuit is performed with a CPLD. The experimental results show the dynamic gate current detection at $10 \mathrm{~mA}(100 \mathrm{mV}$ across $10 \Omega)$ and the device shut down in less than $150 \mathrm{~ns}$. The waveforms are presented in fig. 2(b). This protection can also handle type II "FUL" in much less than Tsc/2, making it a fast and attractive operational gate-driver. This type of fault operation is not described in this article.

(a)

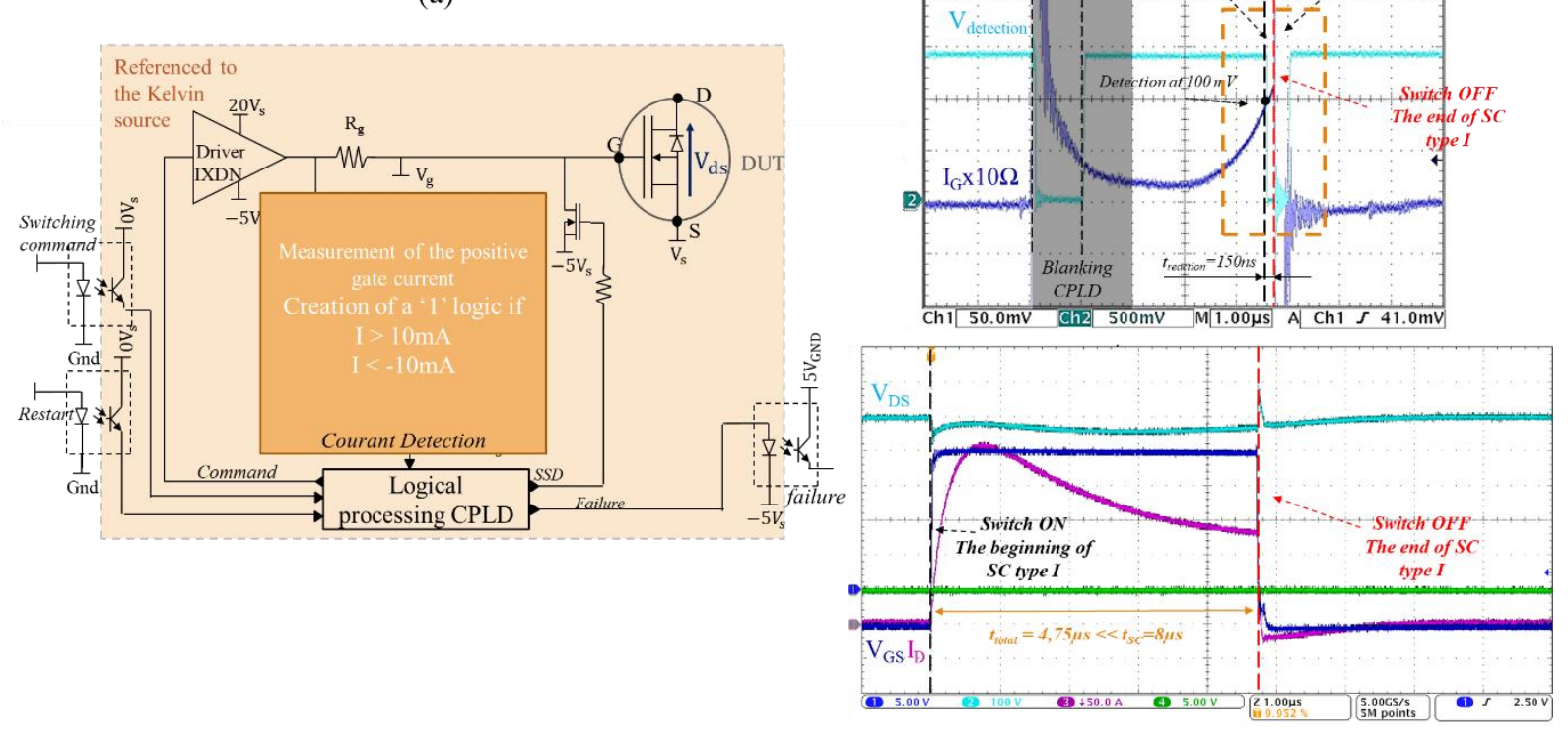

Fig. 2: (a). Simplified detection method using the dynamic gate leakage current and protection circuit. (b). Oscilloscope waveforms of the DUT under protection

\section{Detection using gate charge method under Hard Switch Fault}

Distinctively from the previous method which is not ultra-fast in type I HSF mode but robust in term of SNR criterion, the second method studied in this article is fast and can support high SNR. The method is not based on the gate leakage current monitoring, but still in the gate topology. The studied method depends on the fast integration of the gate current, the gate charge sequence at turn-on. The gate charge method already proved interesting results for IGBTs [11] but unclear results for SiC MOSFETs in terms of performances and practical PCB integration [12]. This study validates experimentally the gate charge method and fault-management for SiC MOSFETs using SMD (Surface-mount technology) components and embedded digital circuit in real full-voltage operation. In the literature there are different architectures proposed for the gate charge method. In this study the circuit is quite the same in the concept. This gate charge method does not require high voltage diode for sensing or setting a detection period as the desaturation method. Moreover, this method has also, potentially, a high SNR. In the principle, this method requires a resettable integrator to estimate the amount of gate charge $\mathrm{Q}_{\mathrm{g}}$; and two comparators. The first comparator is to distinguish the difference between the charge amount under short circuit $\mathrm{Q}_{\mathrm{g}-\mathrm{SC}}$ and normal turn-on (NTO) $\mathrm{Q}_{\mathrm{g}-\mathrm{NTO}}$; the second 
comparator comes to create a reading flag using only a $\mathrm{v}_{\mathrm{gs}}$ signal threshold crossing [13] and not a blanking time is required, fig. 3 .

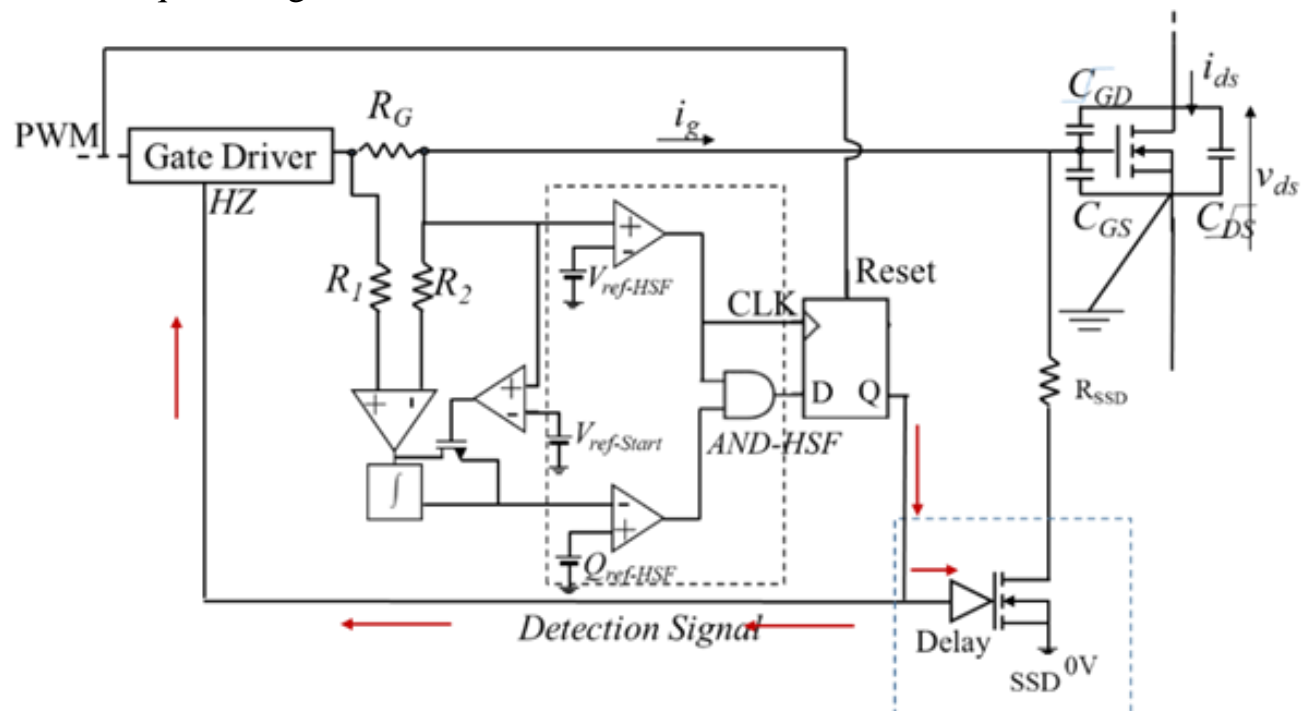

Fig. 3: Basic schematic of the detection and protection circuit using $\mathrm{Q}_{\text {gate }}$ monitoring.

The gate charge in function of $\mathrm{v}_{\mathrm{GS}}$ under NTO and SC-HSF was studied by using fundamental equations and by Simulation using LT-Spice models, then compared to the datasheet, fig. 4(a). This 2D diagram is well known on the study of the gate charge method, offers two important detection keys: the detection zone, and the ratio $\mathrm{S}=\mathrm{Q}_{\mathrm{g}-\mathrm{SC}} / \mathrm{Q}_{\mathrm{g}-\mathrm{NTO}}$.

In order to validate the gate charge method, an experiment was conducted for the gate charge monitoring method. The circuit in fig.4(b) was proposed using on the low side a $1.2 \mathrm{kV}-80 \mathrm{~m} \Omega$, C2M0080120D SiC MOSFET transistor, and on the high side a SiC 600V SDB (Shottky barrier diode) with a load coreless inductor of $270 \mu \mathrm{H}$ for the normal operation. A copper short-strap across the high side is used in order to get the HSF mode, fig. 4(b) is used. The gate driver used is a 3-state fast buffer, allows a high impedance configuration. The SMD circuit was implemented in the circuit and validated, Fig. 5. The power test bench used has for potential reference the kelvin source of the DUT. Low voltage signals (as $\mathrm{V}_{\mathrm{GS}}$ and $\mathrm{V}_{\mathrm{Drv}}$ ) are mostly measured with self-compensated voltage probes (300 V and $1 \mathrm{GHz}$ bandwidth (BP) Tektronix TPP1000). High voltages (as $\mathrm{V}_{\mathrm{DS}}$ ) is typically measured with a probe $(1000 \mathrm{~V}$ and $800 \mathrm{MHz}$ BP Tektronix TPP0850). The current is measured through a $2 \mathrm{GHz}$ coaxial shunt.

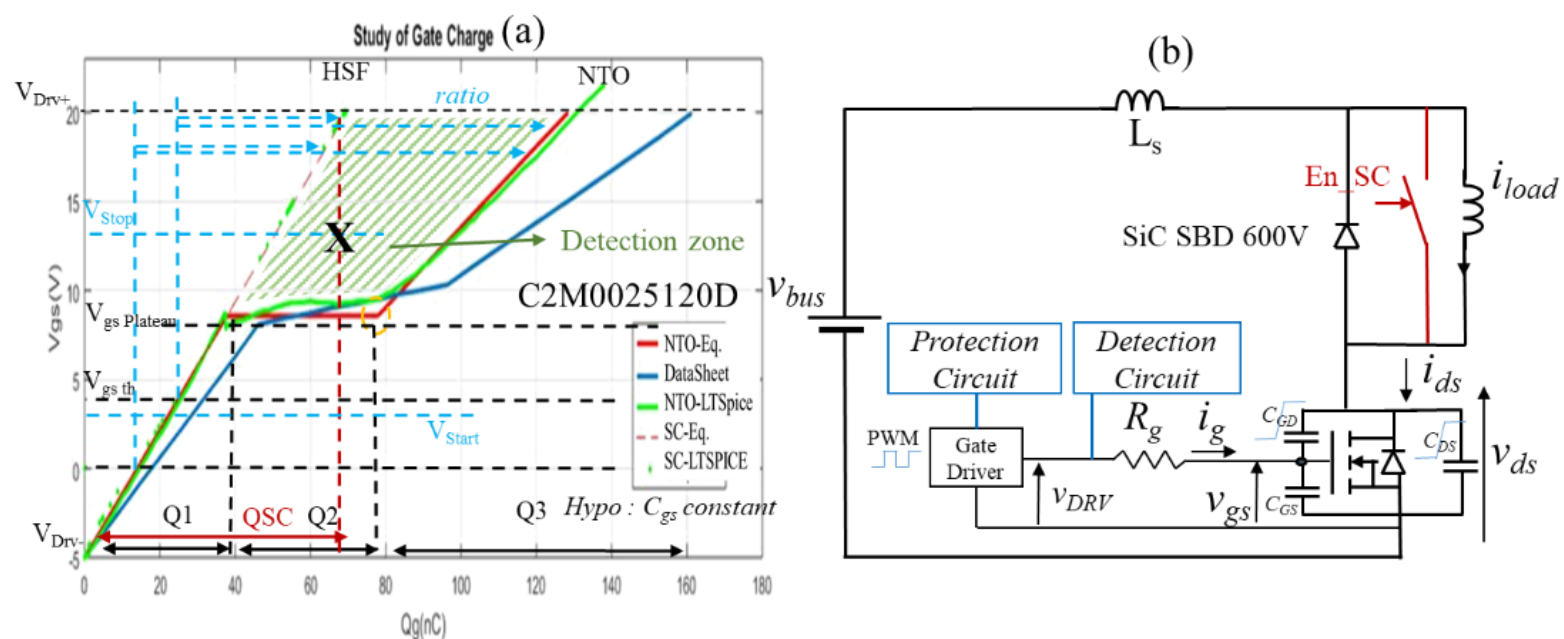

Fig. 4: (a) 2D diagram of the gate charge method. (b) Circuit under test.

The circuit was validated under different $V_{\text {bus }}$ values between $(0-400 \mathrm{~V})$. In fig. 6 \& fig. 7 the experiment was under $\mathrm{V}_{\text {bus }}=400 \mathrm{~V}$ with a $\mathrm{R}_{\mathrm{g}}=10 \Omega$. Fig. 6 presents oscilloscope waveforms under 
NTO, fig. 6(a) gate and drain NTO waveforms. Under NTO the amount of the gate charge is higher than under SC, which activate $\mathrm{Q}_{\mathrm{g}}$ comparator, as shown in fig. 6(b), this activation will be scanned at the arrival of the $\mathrm{V}_{\mathrm{gs}}$ comparator, therefore, the flag remains low. In the other hand; fig. 7(a) shows the short-circuit effect (no drain-source dv/dt) on the drain and gate waveforms, the circuit is under SC, hence the amount of the gate charge is lower than NTO, the $\mathrm{Q}_{\mathrm{g}}$ comparator remains low, which at the arrival of the $\mathrm{V}_{\mathrm{gs}}$ comparator activate the short-circuit detection flag. Both figures were taken using the initial porotype board, on odder to present internal logic signals. The final board presented in Fig.5 does not include internal logic signal.

For a selective detection between NTO vs SC and a better ratio S, the integration can be controlled by adding another threshold level to start the integration. Unfortunately, this reduces the integrator output level, gives less robustness range for other power components and a trade-off must be reached.

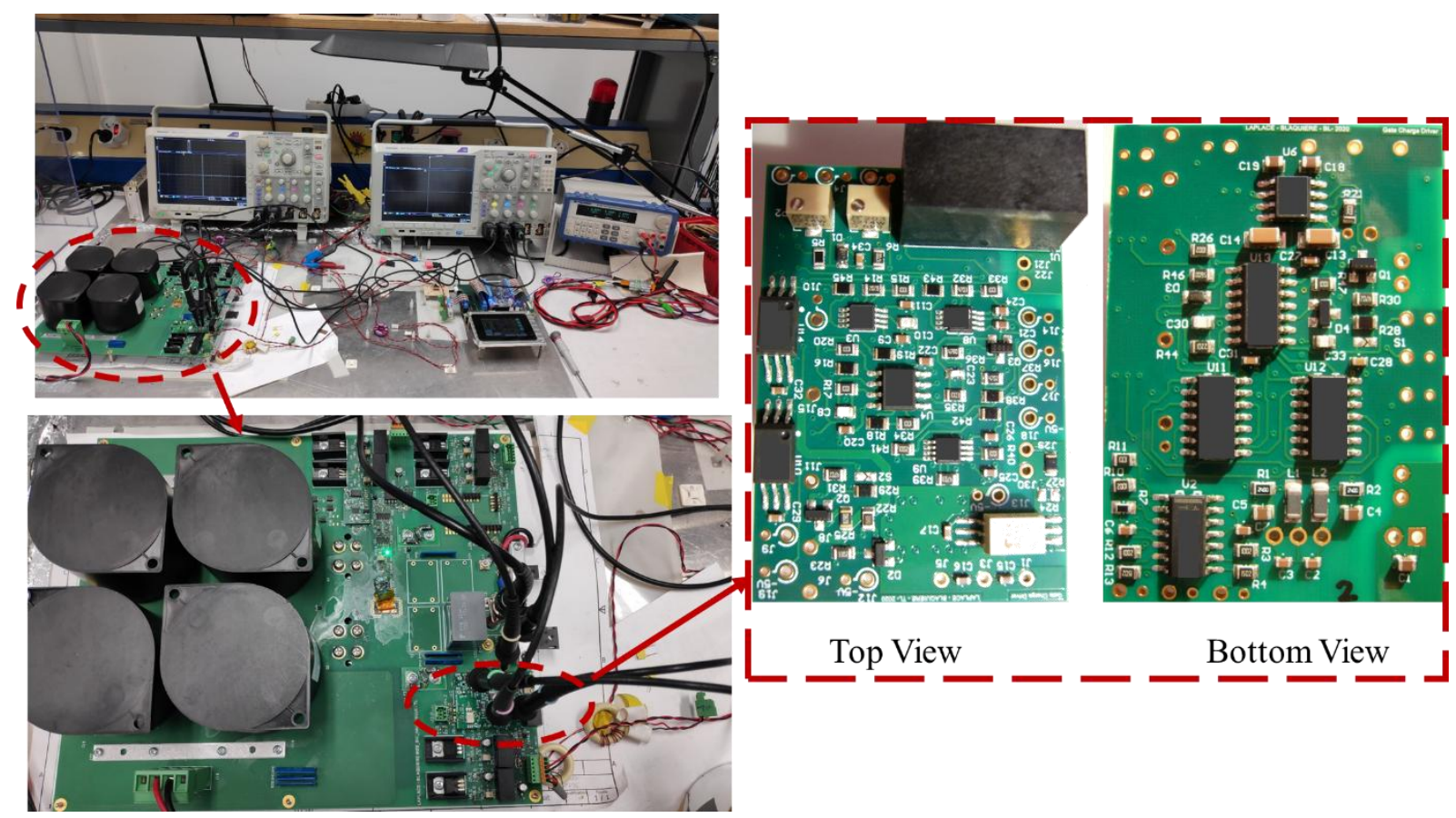

Fig.5: The double-pulse / short-circuit test bench and the SMD board based on the gate charge detection method: 6-layer $19 \mathrm{~mm}^{2} \mathrm{PCB}$ as a safe gate-driver plugging-option.

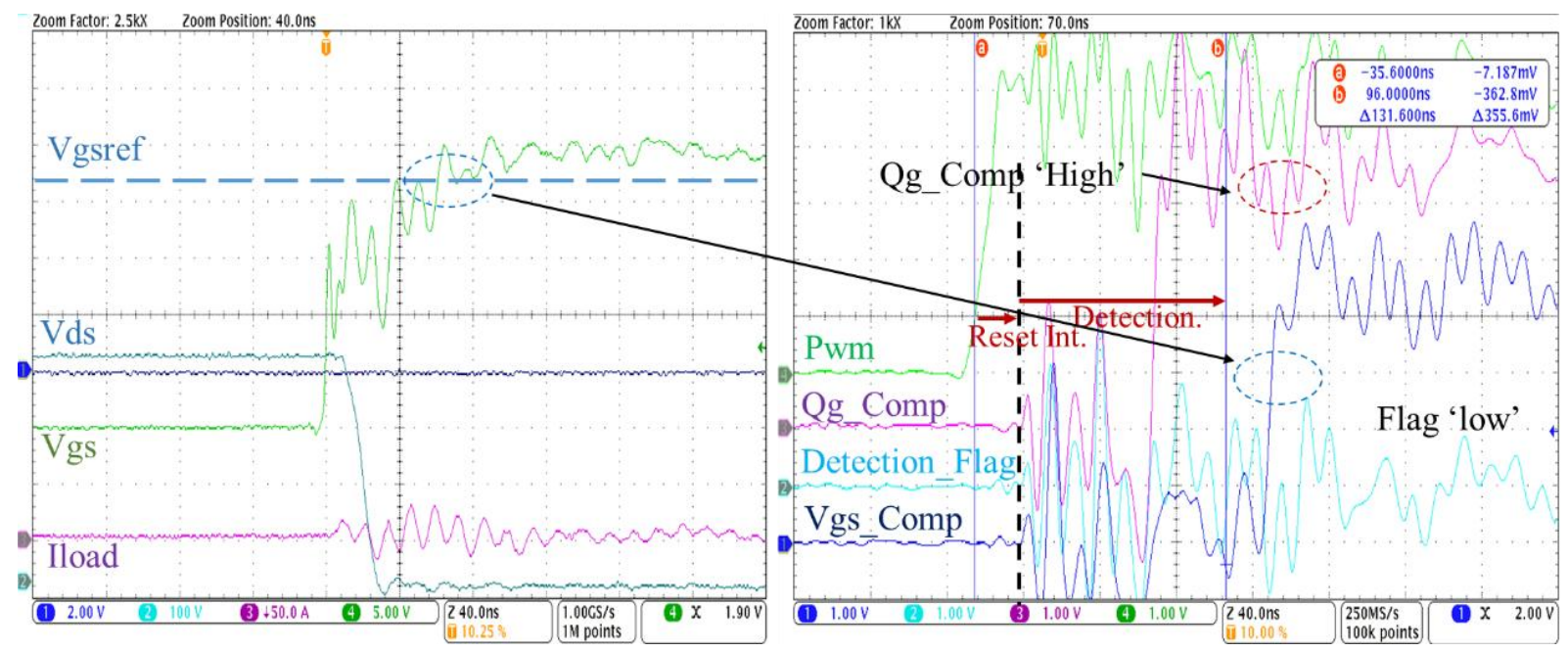

Fig. 6: Oscilloscope waveforms under NTO (Initial prototype) @ 400V $-\mathrm{R}_{\mathrm{G}-\mathrm{Ext}}=10 \Omega-\mathrm{V}_{\text {Supply-Drv }}=-$ 5/20V C2M0080120D 


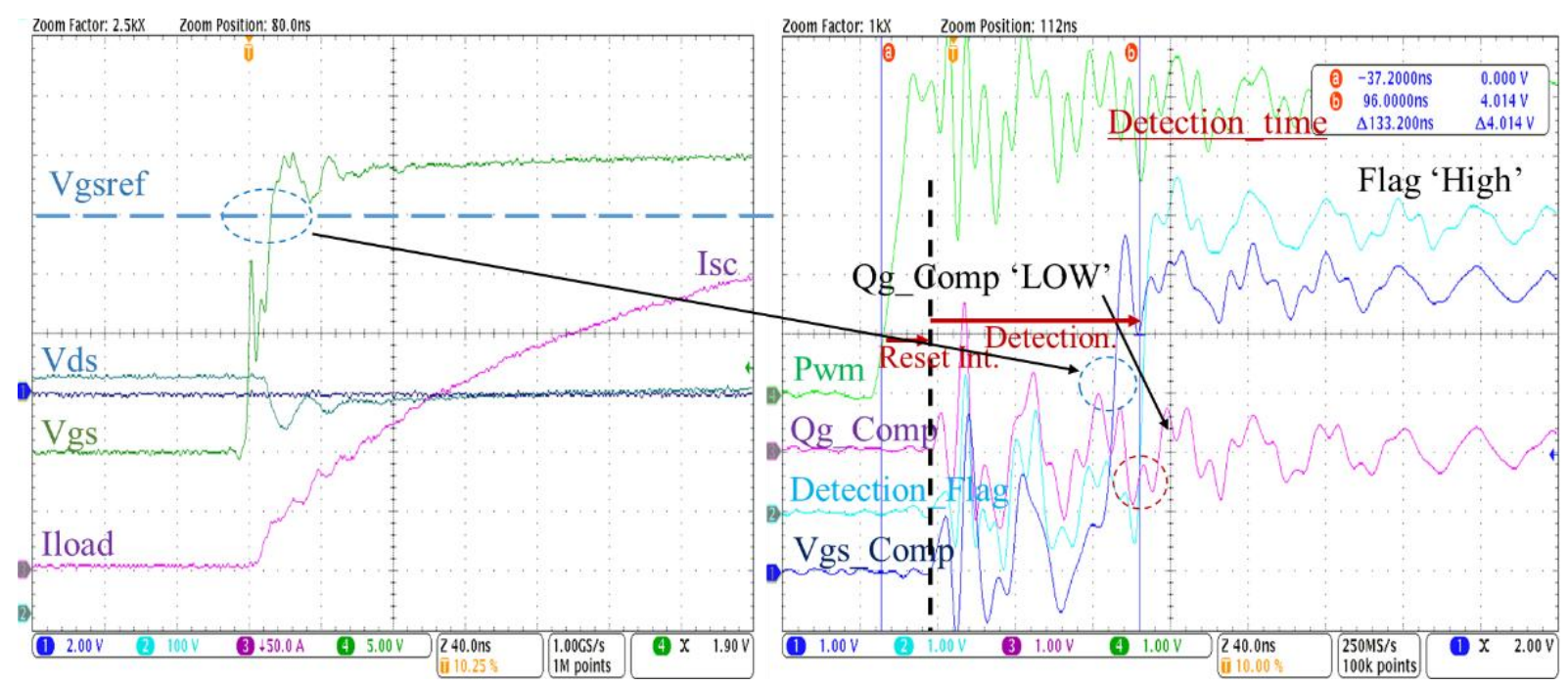

Fig. 7: Oscilloscope waveforms under 400V SC event. (Initial prototype) @ 400V - $\mathrm{R}_{\mathrm{G}-\mathrm{Ext}}=10 \Omega$ $\mathrm{V}_{\text {Supply-Drv }}=-5 / 20 \mathrm{~V}$ C2M0080120D

Fig.8 presents the gate charge quantity under SC-HSF and NTO for both components (gen.II) C2M00800120D device and (gen.III) C3M0065090D device respectively, Fig.8.a and Fig.8.b. As the one can see, the quantity under NTO and SC-HSF for each component is nearly proportional to $50 \%$. The major difference between both component is the total gate charge and the input capacitance of the power device $\mathrm{C}_{\text {iss }}=1.17 \mathrm{nF}, \mathrm{C}_{\text {iss }}=0.76 \mathrm{nF}$. Another important difference is the gate driver supply $\mathrm{V}_{\text {Supply- }}$ Drv $=-5 / 20 \mathrm{~V}, \mathrm{~V}_{\text {Supply-Drv }}=-4 / 15 \mathrm{~V}$. Then, in order to safely protect the device and insure the detection, both references set $\left(\mathrm{Q}_{\mathrm{Ref}}, \mathrm{V}_{\mathrm{Ref}}\right)$ should adapt to the components. The goal of this study is to validate the robustness of the method. Moreover, future studies can be done in order to implement adapted threshold levels.

From fig.8, the immunity band IB is equal $\left|\mathrm{Q}_{\mathrm{Ret}}-\mathrm{Q}_{\mathrm{G}}\right| / \mathrm{Q}_{\text {Ref, }}$, presented in detail in table1. The ratio $\mathrm{S}$ is presented as well, and compared with the gate charge (C) from the datasheet. The difference between the ratio from the datasheet and the measured ratio in voltage (the output of the integrator), is due to the reset added to the integrator to reduce the stress in the beginning of the integration. All the data seems to converge to the pre-study of the method.

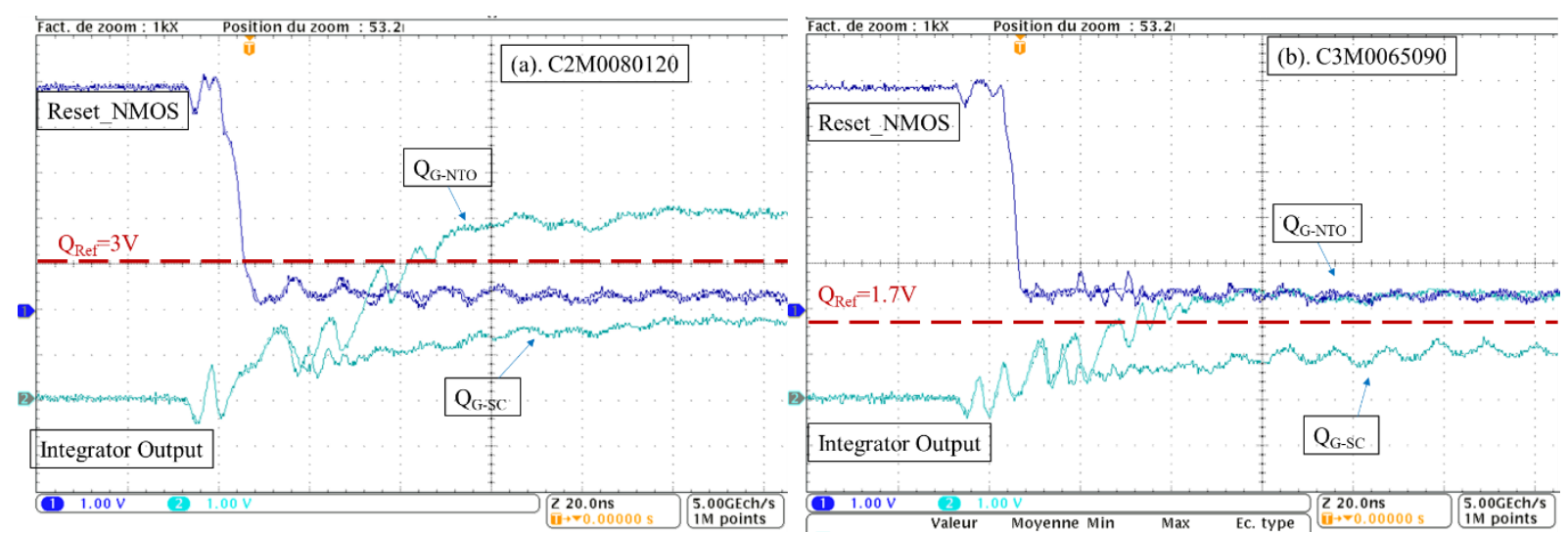

Fig.8. Integrator output " $\mathrm{Q}_{\mathrm{G}}$ " under 400V NTO and SC operation for two power components, (a).C2M0080120D (b).C3M0065090D @ $\mathrm{R}_{\mathrm{g} \_ \text {ext }}=10 \Omega$.

Fig.9. presents an image of $\mathrm{V}_{\mathrm{GS}}$, which represents the voltage $\mathrm{V}_{\mathrm{GS}}$ adapted to $5 \mathrm{~V}$ supply functions, therefore this voltage is compared with a reference level $\mathrm{V}_{\text {Ref }}$ higher than the Miller plateau. This configuration set of the reference will activate the detection. Since the gate switch voltage between both transistor of different generations (G2 \& G3) differ, respectively $-5 / 20 \mathrm{~V}-4 / 15 \mathrm{~V}$, the reference level should adapt for each generation (and not each transistor). 
Table I: Gate charge Immunity Band and Ratio S

\begin{tabular}{|c|c|c|c|}
\hline \multicolumn{2}{|c|}{ Component } & C2M0080120D & C3M0065090D \\
\hline \multicolumn{2}{|l|}{$\mathrm{Q}_{\mathrm{G}-\mathrm{NTO}}$} & $4.2 \mathrm{~V}$ & $2.3 \mathrm{~V}$ \\
\hline \multicolumn{2}{|l|}{$\mathrm{Q}_{\mathrm{G}-\mathrm{SC}-\mathrm{HSF}}$} & $1.8 \mathrm{~V}$ & $1 \mathrm{~V}$ \\
\hline \multicolumn{2}{|l|}{$\mathrm{Q}_{\text {Ref }}$} & $3 \mathrm{~V}$ & $1.7 \mathrm{~V}$ \\
\hline \multirow{2}{*}{$\mathrm{IB}=\left|\mathrm{Q}_{\mathrm{Ref}}-\mathrm{Q}_{\mathrm{G}}\right| / \mathrm{Q}_{\mathrm{Ref}}$} & NTO & 0.4 & 0.35 \\
\hline & $\mathrm{SC}$ & 0.4 & 0.41 \\
\hline \multicolumn{2}{|c|}{$\mathrm{S}_{\%}=\mathrm{Q}_{\mathrm{G}-\mathrm{SC}-\mathrm{HSF}} / \mathrm{Q}_{\mathrm{G}-\mathrm{NTO}}(\mathrm{V} / \mathrm{V})$} & $43 \%$ & $42 \%$ \\
\hline \multicolumn{2}{|c|}{$\mathrm{S}_{\mathrm{DS} \%}(\mathrm{nC} / \mathrm{nC})$} & $34 / 71=>48 \%$ & $17.5 / 35=>50 \%$ \\
\hline
\end{tabular}
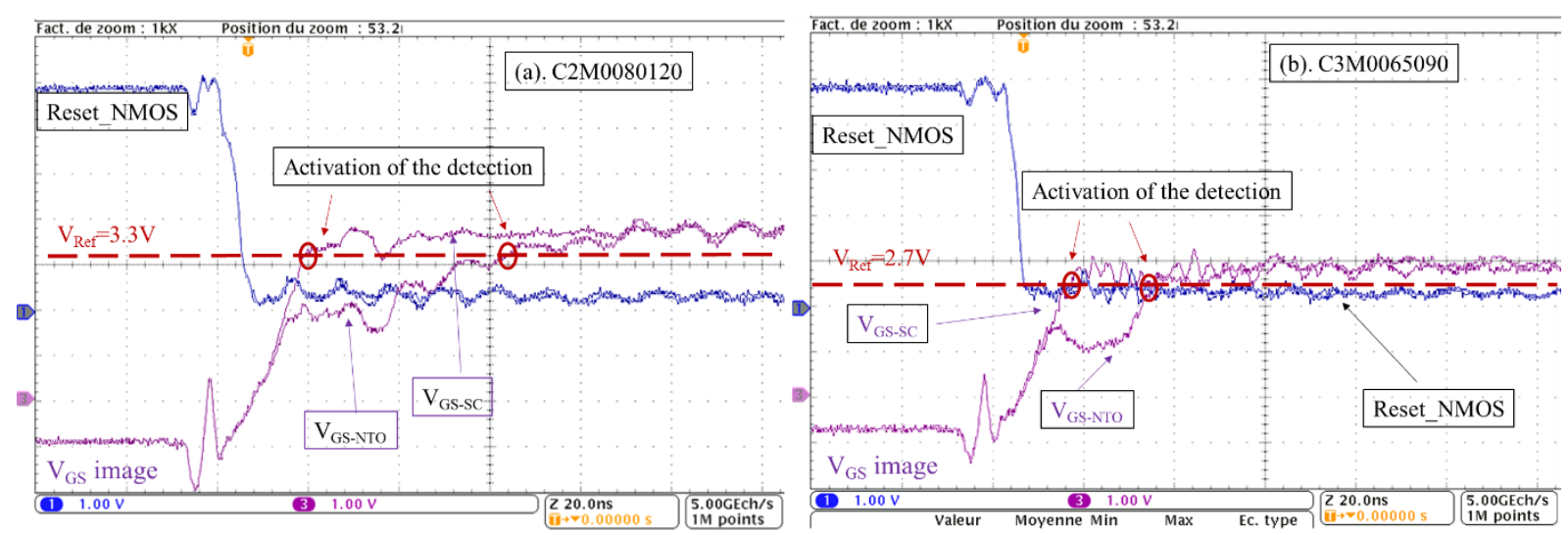

Fig.9. "V $\mathrm{V}_{\mathrm{GS}}$ image" under 400V NTO and SC operation for two power components, (a).C2M0080120 (b).C3M0065090 @ $\mathrm{R}_{\mathrm{G}_{-} \text {ext }}=10 \Omega$.

The detection flag has been activated after 133ns including the buffer propagation time $10 \mathrm{~ns}$ with the initial prototype and $118 \mathrm{~ns}$ with the final board. The detection time does not depend only on how fast our system is, the detection time depends obviously on the value of $R_{\mathrm{g}}$, the input capacitance of the power device, slew rate $d v_{\mathrm{gs}} / \mathrm{dt}$ and how low the reference level is defined $\left(>\mathrm{V}_{\mathrm{gsm}}\right)$, etc... The slew rate between NTO vs SC change as presented in fig. 6-7, the $\mathrm{V}_{\mathrm{gs}}$ comparator under NTO arrives after SC, which is a positive thing to detect faster. Same as the reference levels, the threshold gate level was defined at a robust level $18 \mathrm{~V}$, with a lower level the detection will be earlier but with a trade-off on the SNR.

\section{Protection of the device}

After the detection of the fault, the SiC MOSFET must be turned off safely, to stop $\mathrm{I}_{\mathrm{ds}}$ from increasing and to avoid a dangerous over-voltage. Therefore, to protect the circuit safely, a soft shut down SSD system is included in the SMD. If the protection is not well designed it can lead to breakdown voltage due to high negative $\mathrm{dI}_{\mathrm{ds}} / \mathrm{dt}$ caused by the total stray inductance, the protection should be soft to prevent this phenomenon.

After activation of the D flip-flop (Detection flag), the 3-state fast driver output is turned under high impedance (HZ), to avoid being short circuited by the SSD circuit. Then, after a delay at least equivalent to the driver propagation time, the SSD is activated, including a serial resistance $\mathrm{R}_{S S D}=75 \Omega$, to turn off softly the device through discharging the gate input capacitance. The delay has been chosen 
to be set at 50ns, for safety, fig.10. The observed plateau in the gate-source voltage $\mathrm{V}_{\mathrm{GS}}$ under protection is due to the inductive effect through the kelvin inductance $\mathrm{L}_{\mathrm{SK}}$ (parasitic inductance of package and terminal pin on PCB in the presence of a di/dt $<0$ ).

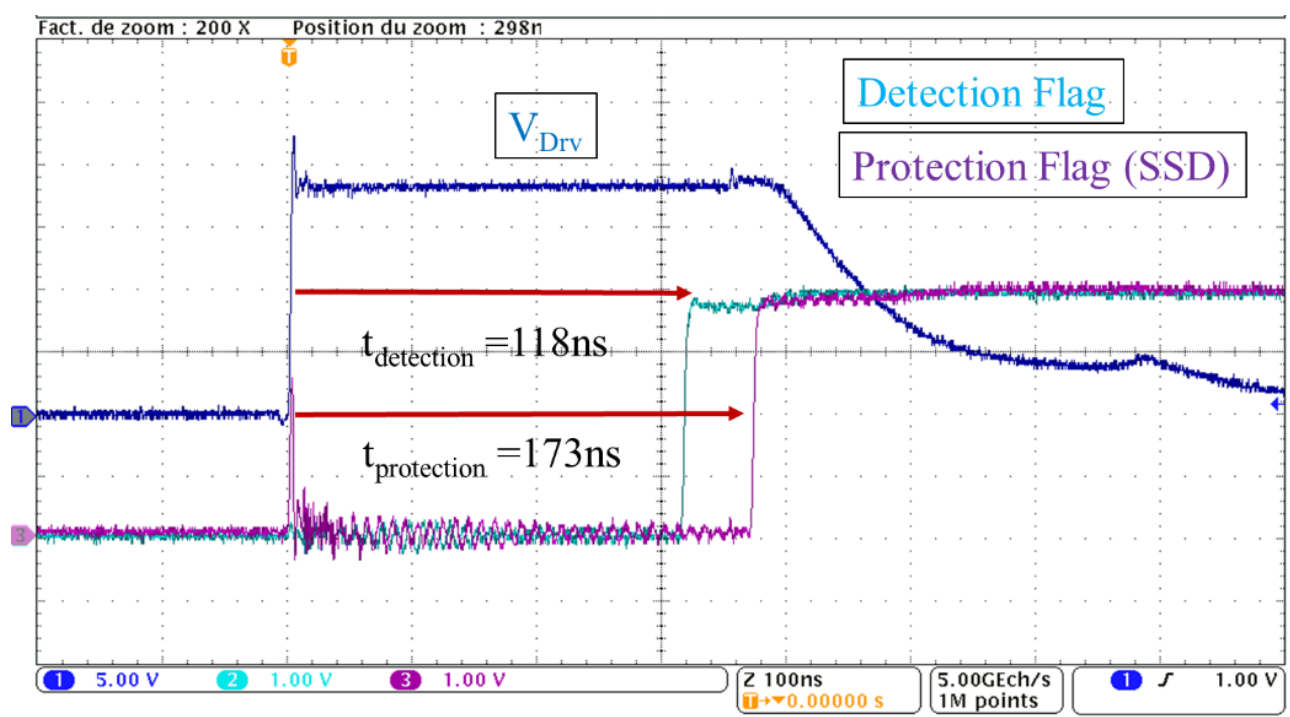

Fig.10: Logic Output signal, representing detection and protection time, C2M0080120 DUT, @ $400 V-R_{\mathrm{g} \_ \text {ext }}=10 \Omega$.

Fig. 11 presents the waveforms of the device under short circuit, including protection. After the detection flag, the buffer is putted under $\mathrm{HZ}$ and the SSD is activated, the $\mathrm{V}_{\mathrm{GS}}$ starts to softly decrease for 530ns to reach $\mathrm{V}_{\text {Drv- }}(-5 \mathrm{~V}$ or $-4 \mathrm{~V})$, limiting $\mathrm{I}_{\mathrm{ds}}$ to go higher. The $\mathrm{V}_{\mathrm{GS}}$ comparator goes back to low but the flag remains high until the PWM of the buffer goes low, allowing the system to detect at the next PMW pulse.

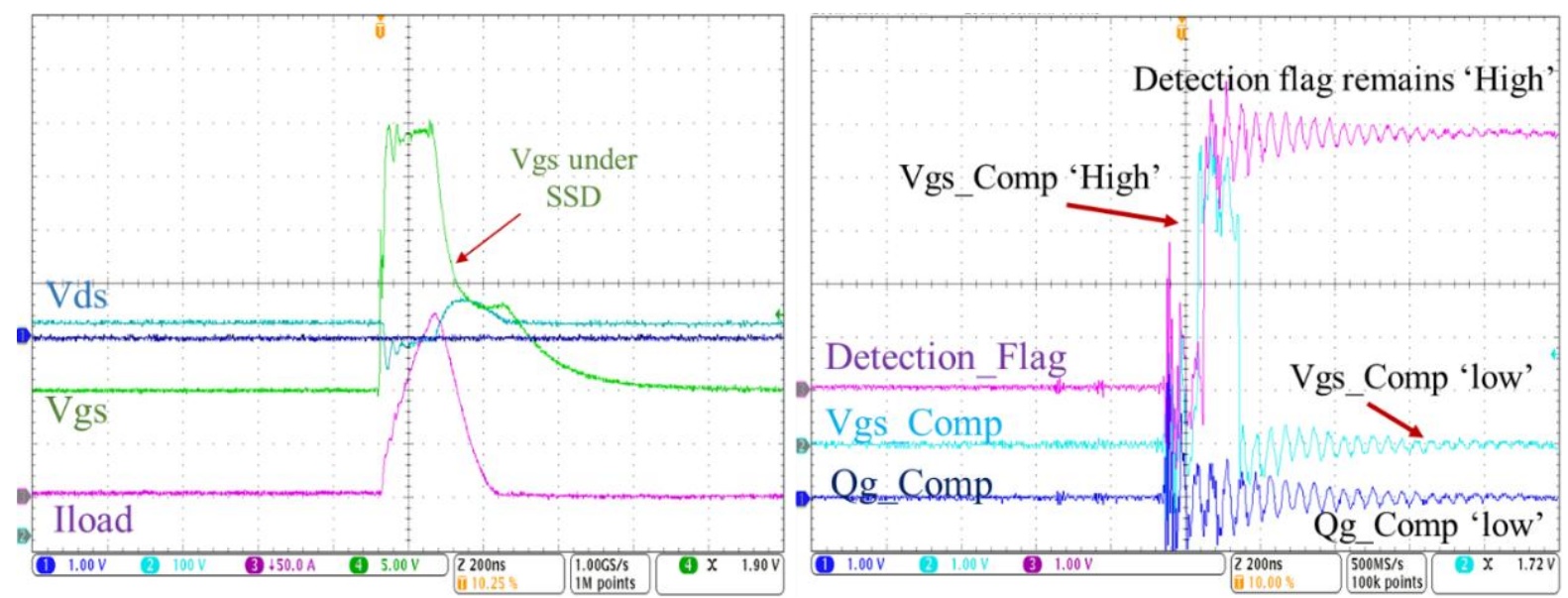

Fig.11: Oscilloscope waveforms, Circuit under SSD protection (SMD first prototype) C2M0080120 DUT @ $400 V-R_{\mathrm{g} \_ \text {ext }}=10 \Omega-\mathrm{R}_{\mathrm{SSD}}=75 \Omega$.

The high speed detection allows safety turned off at a current level well below the saturation current of the channel. Indeed, the turn-off is done here at 175A while the saturation current is 290A. The SSD could therefore be accelerated in order to further reduce the energy stress at turn-off detection for the $\mathrm{SiC}$ MOSFET and to preserve its capability of endurance to the short-circuit cycles that the device could have to undergo throughout its life in accidental conditions of use. Fig. 12 presents the protection behavior under 2 pulse. The behavior is observed under $400 \mathrm{~V}$, for both operation, protection not included and included. In order to fully cover the robustness of this detection method, the circuit is validated under different bus voltages $(50,150,250,400 \mathrm{~V}$ ) which is lower than the typical $1.2 \mathrm{kV}$ (or $0.9 \mathrm{kV}$ ) operational voltage, which makes the detection between NTO vs SC critical, Fig.13.The one 
can see that with the final prototype the drain SC current is lower and stopped earlier than the previous prototype. The current is limited around 130A.

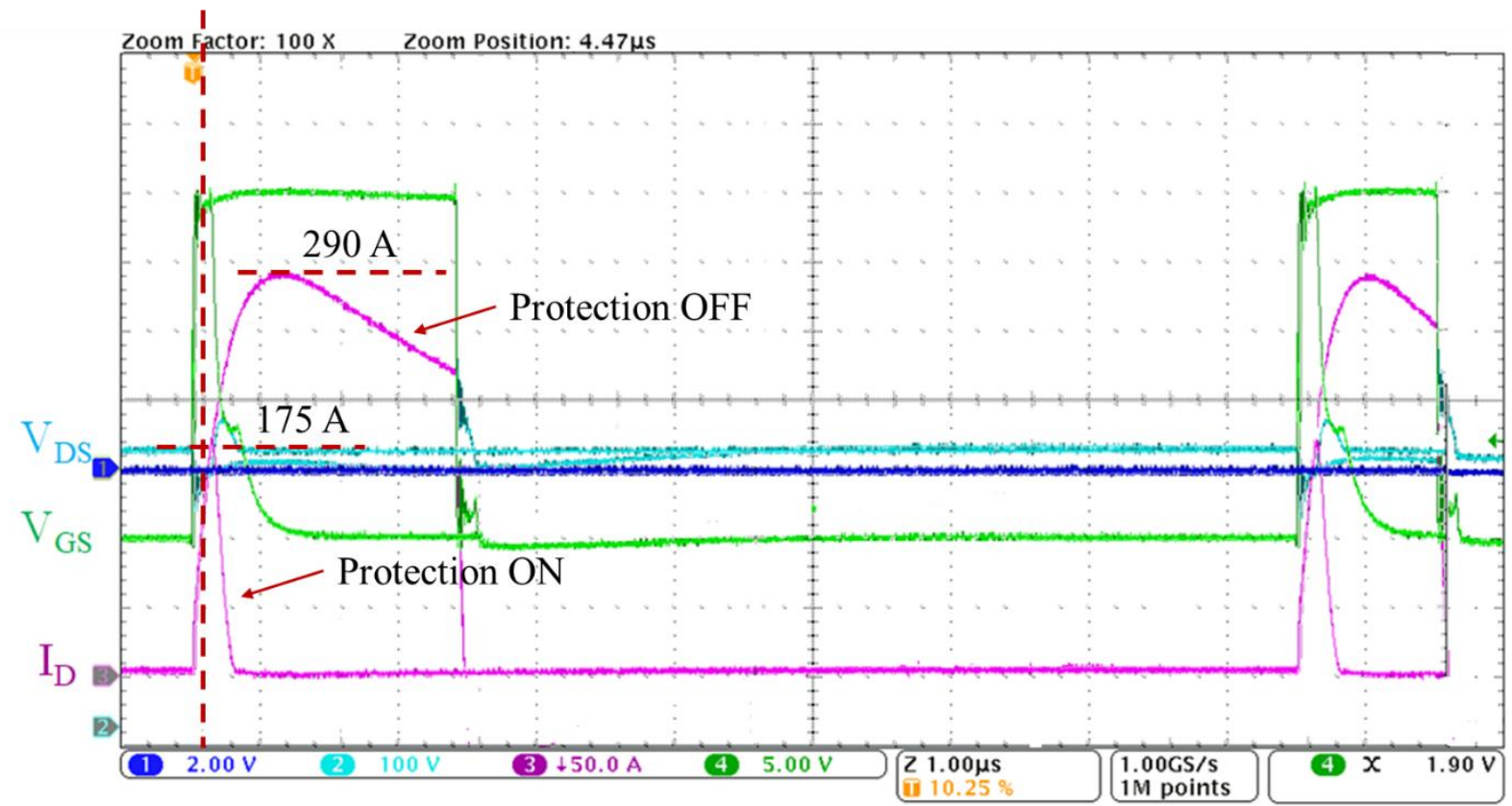

Fig. 12: DUT before and after protection, C2M0080120 DUT @ 400V $\mathrm{R}_{\mathrm{g} \_ \text {ext }}=10 \Omega$. (Initial prototype)

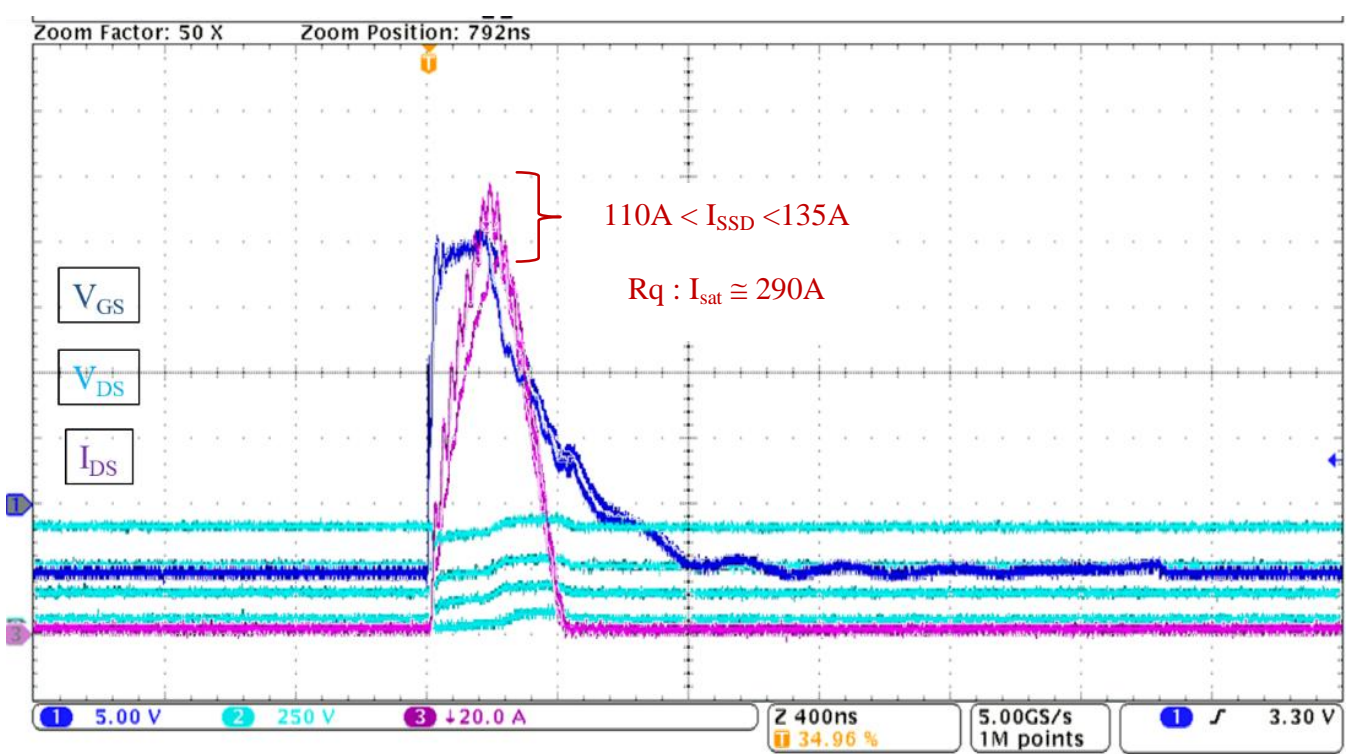

Fig. 13: DUT under protection for different $\mathrm{V}_{\text {Bus }} \in[50,150,250,400 \mathrm{~V}], \mathrm{C} 2 \mathrm{M} 0080120$ DUT, $@ \mathrm{R}_{\mathrm{g} \_\mathrm{ext}}=10 \Omega$.( last prototype)

\section{Conclusion}

2D diagnosis once again proves it is the perfect candidate for short circuit detection and protection. In a first place, the gate charge method (2D diagnosis) detects as fast as possible the behavior of the circuit, within $118 \mathrm{~ns}$. In the second place, the gate leakage current method, comes to confirm and support the detection of the short-circuit, before the failure of the power transistor. With those experiments both methods were validated. Robustness study is led for different components and $\mathrm{V}_{\text {Bus }}$ values. Studies are led to finalize FUL detection in other specific mode not presented in this paper. Moreover, the detection circuit can be further optimized and the delays can be strongly reduced. At the end, the goal is to integrate all the functions in a dedicated gate driver Integrated Circuit in CMOS technology, for fast detection. It is important to note that with such a fast protection, the fault current 
is limited to $135 \mathrm{~A}$ against nearly $290 \mathrm{~A}$ with no protection. In such a short time and at such a low current value, the dynamic temperature of the chip remains lower than the melting temperature of the aluminum to metal of the die, which would allow a large number of repeated short circuits without ageing effect [14]. This last point is one of the future properties to be highlighted in the continuity of this work.

\section{References}

[1] Baliga B.J.: Silicon Carbide Power Devices, World Scientific, 2005.

[2] Wang Z., et al.: Design and Performance Evaluation of Overcurrent Protection Schemes for Silicon Carbide (SiC) Power MOSFETs, IEEE TIEs 61, 5570-5581 (2014).

[3] Romano G., et al.: Influence of design parameters on the short-circuit ruggedness of SiC power MOSFETs, in $201628^{\text {th }}$ ISPSD, juin 2016, p. 47- 50,

[4] Chen J., et al.: A Smart IGBT Gate Driver IC with Temperature Compensated Collector Current Sensing, in IEEE Transactions on Power Electronics, vol. 34, no. 5, pp. 4613-4627, May 2019.

[5] Sadik D., et al.: Short-Circuit Protection Circuits for Silicon-Carbide Power Transistors, in IEEE Transactions on Industrial Electronics, vol. 63, no. 4, pp. 1995-2004, April 2016.

[6] Awwad A. E., Dieckerhoff S.: Short-circuit evaluation and overcurrent protection for SiC power MOSFETs, 2015 17th EPE'15 ECCE-Europe, Geneva, 2015, pp. 1-9.

[7] Bertelshofer T., Maerz A., Bakran M.: Design Rules to Adapt the Desaturation Detection for SiC MOSFET Modules, PCIM Europe 2017, Nuremberg, Germany,

[8] Bakran M., Hain S.: Integrating the New 2D - Short circuit detection method into a power module with a power supply fed by the gate voltage, 2016 IEEE (SPEC), Auckland, 2016, pp. 1-6.

[9] Boige, F., Trémouilles D., Richardeau F.: Physical origin of the gate current surge during short-circuit operation of SiC MOSFET, IEEE Electron Device Lett., pp. 1-1, 2019

[10] Boige F., and Richardeau F.: Gate leakage-current analysis and modelling of planar and trench power SiC MOSFET devices in extreme short-circuit operation, Microelectron. Reliab., Sep. 2017

[11] Oberdieck K., Schuch S., DeDoncker R. W.: "Short circuit detection using the gate charge characteristic for Trench/Fieldstop-IGBTs", EPE'16 ECCE Europe, Karlsruhe, 2016, pp. 1-10.

[12] Horiguchi T., Kinouchi S., Nakayama Y., Akagi H.: A fast short-circuit protection method using gate charge characteristics of SiC MOSFETs, 2015 IEEE ECCE, Montreal, QC, 2015, pp. 4759-4764.

[13] Barazi Y., Rouger N., Richardeau F.: Comparison between ig integration and vgs derivation methods dedicated to fast short circuit 2D diagnosis for wide band gap power devices, Mathematics and Computers in Simulation, 2020.

[14] Fayyaz A., Boige F., Borghese A., Guibaud G., Chazal V., et al.: Aging and failure mechanisms of SiC Power MOSFETs under repetitive short- circuit pulses of different duration, ICSCRM 2019, Japan 